\title{
The Effects of Hamstring Stretching on Leg Rotation during Knee Extension
}

\author{
Atsushi Kimura, PT ${ }^{1)}$ \\ 1) Department of Rehabilitation, Medical Education and Research Center, Meiji University of \\ Integrative Medicine: 6-1 Honoda Hinotani, Hiyoshi-cho, Nantan-city, Kyoto 629-0392, Japan. \\ TEL +81 771-72-1181, FAX: +81 771-72-0326.
}

\begin{abstract}
Purpose] This study investigated the effects of hamstring stretching on leg rotation during active knee extension. [Subjects] Subjects were 100 bilateral legs of 50 healthy women without articular disease. [Methods] Hamstring hardness, leg rotation and muscle activities of the knee extensors during active knee extension were measured before and after hamstring stretching. [Results] Hamstring hardness was significantly decreased after hamstring stretching. The leg rotation angle, variation in leg rotation angle, variation in leg external rotation angle, and muscle activities of the vastus lateralis and rectus femoris were significantly increased after hamstring stretching. A moderate positive correlation was found between variation in leg rotation and variation in muscle hardness in hamstring. [Conclusion] Leg rotation during active knee extension was increased by hamstring stretching. Hamstring stretching would be effective as a pretreatment for restoring proper leg rotation when knee extension is conducted as a therapeutic exercise.
\end{abstract}

Key words: Hamstring stretching, Leg rotation

(This article was submitted Dec. 28, 2012, and was accepted Jan. 31, 2013)

\section{INTRODUCTION}

Knee extension is conducted as a therapeutic exercise for dysfunction of the knee joint and includes passive exercise, active assistive exercise, active exercise, and active resistive exercise $^{1)}$. Passive exercise is generally conducted when the results of manual muscle testing of the quadriceps femoris muscles are extremely poor; active assistive exercise is conducted when the results of the testing are poor; active exercise is conducted when the results are fair; and active resistive exercise, in which loads such as weights are applied, is conducted when the results are better than fair. Passive exercise involves passively having the lower leg moved against the femur with the knee joint as a fulcrum. On the other hand, active assistive exercise, active exercise, and active resistive exercise are based on the third class lever of the quadriceps femoris muscle as the force, the knee as a fulcrum, and the weight load of the lower leg and foot as the load ${ }^{1)}$. Because each type of exercise involves mechanical load being applied to the knee joint, which serves as a fulcrum, it is essential that knee joint motion should be kinematically proper during exercise.

The knee joint possesses two degrees freedom: flexion and extension, and the resulting leg rotation. Leg rotation is found in the full range of motion of flexion and extension, with leg rotation in the direction of internal rotation accompanying flexion, and leg rotation in the direction of external rotation accompanying extension ${ }^{2-4)}$. Leg rotation increases in the direction of external rotation in the range

E-mail: a_kimura@meiji-u.ac.jp from $30^{\circ}$ flexion to final extension which is the final phase of extension: this is called the "screw-home movement"5).

The screw-home movement is caused by differences in size between the medial and lateral condyles of the femur, strain in the anterior cruciate ligament, and external traction exerted by the quadriceps femoris muscle against the tibia, and it is important for gaining dynamic stability in the knee joint ${ }^{2}$. Sugawara et al. ${ }^{6}$ ) reported failure of leg rotation in knee osteoarthritis patients, indicating the importance of establishing a method for restoring leg rotation. Their findings indicate the importance of leg rotation in knee joint extension as a therapeutic exercise for dysfunction of the knee joint. When leg rotation does not arise from knee joint extension, we must investigate the factors limiting it. We may then conduct knee extension after restoring proper leg rotation by implementing a therapeutic approach for the limiting factors. However, while there have been many reports which have analyzed leg rotation ${ }^{7-13)}$, there have been few on the causes of the failure of leg rotation and how it might be restored.

The hamstring, the antagonist of knee extension, is made up of the semitendinosus muscle, the semimembranosus muscle, and the biceps femoris muscle. The semitendinous muscle and the semimembraneous muscle form the medial hamstring, while the biceps femoris muscle forms the lateral hamstring. Both originate in the ischial tuberosity. The medial hamstring muscle fibers attach themselves to the anterior of the medial condyle of the tibia forming the pes anserinus, while the lateral hamstring muscle fibers attach themselves to the posterior surface of the head of the fibula $^{2}$. As these are bi-articular muscles, knee extension occurs by bringing the origin and insertion of the hamstring 
together, by reducing the flexion angle of the hip joint with retroversion of the pelvis when the knee is extended in the sitting position. When the flexion angle of the hip joint does not change, the knee is extended while stress is applied to the "joint play" region within the knee joint, lowering the tibia which is the origin of the hamstring. If the hamstring is stretched from resting length by knee extension when there is reduced extensibility of the hamstring, defensive contraction induced by stretch reflex occurs, inhibiting the knee antagonizing extensors and reducing their activity. This indicates that when hamstring extensibility is reduced during knee extension, the resultant leg rotation may be limited. However, it is not clear whether changes in hamstring extensibility affect leg rotation during knee extension. Accordingly, this study was conducted to clarify the effects of hamstring stretching on leg rotation during knee extension.

\section{SUBJECTS AND METHODS}

The subjects were 100 bilateral legs of 50 healthy women without articular disease. The mean age of the subjects was $20.9 \pm 1.4$ years, their mean weight was $54.7 \pm 10.3 \mathrm{~kg}$ and their mean height was $158.3 \pm 6.4 \mathrm{~cm}$. Subjects' consent was obtained after sufficient explanation was given regarding the aim of the study, management of personal information obtained in the study, and the right of the subjects to withdraw from the study at any time if they wished to do so. The study was conducted after obtaining approval from the Research Ethics Committee of Meiji University of Integrative Medicine (authorization number 21-88).

The study protocols are described below. The muscle hardnesses of the bilateral medial and lateral hamstrings of the subjects were measured. Leg rotation with active knee extension was measured using a 3-dimensional motion analysis system, and muscle activities of the knee extensors were measured by the surface electromyograph. For the leg with greater muscle hardness in the medial hamstring, direct stretching was conducted through intervention for the medial and lateral hamstrings (the "stretching group"). Meanwhile the other leg was considered to be at rest (the "control group"). After the intervention, the same measurements as prior to the intervention were performed, and the results were compared with those of prior to the intervention. We also examined the correlation between changes in muscle hardness of the medial and lateral hamstrings and leg rotation, and muscle hardness of the medial and lateral hamstrings and muscle activities of the knee extensors.

Muscle hardness was measured by a muscle durometer (Axiom biosensor Venustron II, Axiom, Fukushima, Japan). The indenter was placed perpendicularly above the skin at the measurement site using a special arm (Fig. 1). Measurement started when the indenter touched the skin. The indenter was depressed to a depth of $8 \mathrm{~mm}$, then returned to the starting position. The speed of the indenter was set at $3 \mathrm{~mm} / \mathrm{sec}$. The pressure on the indenter at the depth of $8 \mathrm{~mm}$ was measured as the repulsion force of the muscle. The muscle is hard when the pressure is high, and the muscle is soft when the pressure is low. Muscle hardness was measured in the prone position. Measurements were

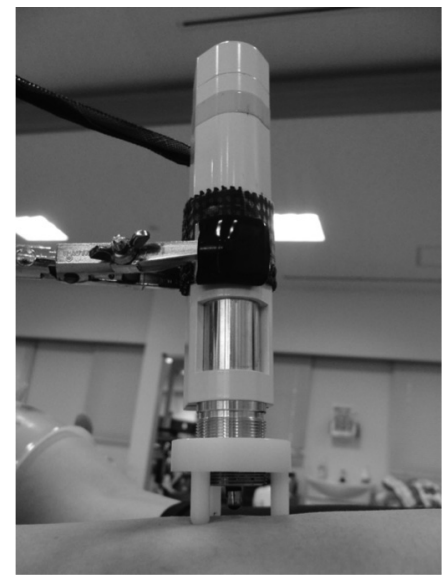

Fig. 1. Measurement of hamstring hardness

taken with 5 degrees of ankle dorsiflexion, 20 degrees of knee flexion, and the hip in the neutral position. A plastic ankle foot orthosis was affixed to both ankles, and a pillow was placed at the distal point of the legs to standardize the positions measured. Measurement sites of the medial hamstring were the muscle belly at the midpoint of the ischial tuberosity and medial condyle of the tibia. Measurement sites of the lateral hamstring were the muscle belly at the midpoint of the ischial tuberosity and the head of fibula. The measurement sites were marked to ensure the same site was measured before and after the intervention. Measurement was done three times at the same site and the mean value was used as the representative value.

For measurement of leg rotation at the knee, the subjects wore on tight-fitting leggings and 10 reflective markers were bilaterally placed, 5 on each leg, at the mid-thigh, medial knee joint gap, lateral knee joint gap, medial malleolus, and lateral malleolus. Subjects lay supine on a bed with their lower legs hanging down and the femur fixed to the bed by a belt. The tester checked to make sure the subjects' soles were not touching the floor. Active knee extension with the ankle joint at maximal dorsiflexion was conducted from a starting position of 90 degrees knee flexion to full extension of the knee. Subjects' movements were recorded after explaining the task orally and confirming it was understood. Subjects practiced the task once before measurement. The positions of the reflective markers were measured using a 3-dimensional motion analysis system (VICON512, Oxford Metrics, Oxford, UK), and knee joint extension angle and leg rotation angle were calculated by software (Body Builder3.6, Oxford Metrics, Oxford, UK). To ascertain angular variation of the leg rotation angle from the initial position of 90 degree knee flexion, the initial angle was adjusted to 0 degrees internal/external rotation, with displacement in the direction of external rotation shown as positive and displacement in the direction of internal rotation shown as negative. The first phase of extension was defined as going from 90 degrees of knee flexion to 60 degrees of knee flexion, the middle phase from 60 degrees of knee flexion to 30 degrees of knee flexion and the final phase from 30 degrees 
of knee flexion to full extension. The mean leg rotation angle in each phase was calculated as the representative value of each phase. The difference of the minimum and maximum leg rotation angles generated by knee extension from 90 degrees of flexion to full extension were calculated as the variation in leg rotation angle. The maximum leg rotation angle in the direction of external rotation was calculated as the variation in leg external rotation angle while the maximum leg rotation angle in the direction of internal rotation was calculated as the variation in leg internal rotation angle.

Before measurement of the muscle activities of the knee extensors, the measurement site was cleaned with an alcohol wipe. Then, disposable surface electrodes were attached and muscle activities were measured using surface electromyography (MYOSYSTEM1200, Noraxon U.S.A. Inc. Arizona, USA). The target muscles were the rectus femoris, vastus lateralis and vastus medialis. The measurement sites of each muscle were as follows: the midpoint between the patellar superior border and anterosuperior iliac spine on the anterior surface of the thigh for the rectus femoris, the lateral surface of the thigh in a five-finger-wide area from the patellar superior border for the vastus lateralis, and a four-finger-wide area from the patellar superior margin for the vastus medialis ${ }^{14)}$. Using the measurement results, average rectified value (ARV) per second were calculated using an analog-to-digital converter (TRIAS System, DKH, Tokyo, Japan). At the end of the experiment, the maximal voluntary contraction(MVC) of each muscle was measured. Subjects lay supine on a bed with their legs hanging down, and the MVC of each muscle was measured for 5 seconds. ARV of the middle, 3 seconds was calculated. ARV during active knee extension was divided by ARV of MVC to calculate $\%$ MVC for each muscle.

Hamstrings were stretched by direct stretching in which pressure was applied in the direction transverse to the axis of the medial and lateral hamstring per the Matsumoto method $^{15)}$, so as not to induce articular movement of the knee. Direct stretching was conducted in the prone position, with the hip in the neutral position and the knees extended. The muscles were stretched for 30 seconds in 3 places; the proximal and distal muscle-tendon junctions of the muscles, and the centers of their muscle bellies. The stretching was done 3 times with 15 second breaks in between.

We compared hamstring muscle hardness, leg rotation angle, variation in leg rotation angle, variation in leg external rotation angle, variation in leg internal rotation angle, and \% MVC of the rectus femoris, vastus lateralis and vastus medialis between before and after the intervention. The paired t-test was used when normality was established by the Kolmogorov-Smirnov test. The Wilcoxon rank sum test was used when normality was not established. For comparison between the stretching and control groups, the independent t-test was used when the Kolmogorov-Smirnov test established normality, while the Mann-Whitney U-test was used when normality was not established. Pearson correlation coefficients were calculated to investigate the correlations between muscle hardness variation and variation in leg rotation angle, variation in leg external rotation angle and variation in leg internal rotation angle, and between
Table 1. Changes in the muscle hardness

\begin{tabular}{llcl}
\hline & & $\begin{array}{c}\text { Stretching } \\
\text { group }\end{array}$ & $\begin{array}{c}\text { Control } \\
\text { group }\end{array}$ \\
\hline Medial & pre-intervention $(\mathrm{g})$ & $110.9 \pm 24.7$ & $92.0 \pm 19.8^{\dagger \dagger}$ \\
hamstring & post-intervention $(\mathrm{g})$ & $83.2 \pm 15.8^{*}$ & $95.2 \pm 17.5^{\dagger \dagger}$ \\
\hline Lateral & pre-intervention $(\mathrm{g})$ & $107.7 \pm 25.6$ & $97.3 \pm 24.1^{\dagger}$ \\
hamstring & post-intervention $(\mathrm{g})$ & $85.8 \pm 14.9^{*}$ & $96.6 \pm 19.7^{\dagger \dagger}$ \\
\hline
\end{tabular}

Mean $\pm \mathrm{SD},{ }^{*} \mathrm{p}<0.01$, significant difference between pre- and post-intervention

$\dagger \mathrm{p}<0.05$, significant difference between groups

i† $\mathrm{p}<0.01$, significant difference between groups

muscle hardness and variations in the muscle activities of the knee extensors. The data were analyzed using SPSS $11.0 \mathrm{~J}$ for Windows and values of $\mathrm{p}<0.05$ were considered statistically significant.

\section{RESULTS}

Table 1 shows the results for muscle hardness. Mean values and standard deviation of muscle hardness are shown. The hardness values of the medial hamstring in the stretching group were $110.9 \pm 24.7 \mathrm{~g}$ prior to the intervention, and $83.2 \pm 15.8 \mathrm{~g}$ after the intervention, a significantly lower value after the intervention $(\mathrm{p}<0.01)$. In the control group, the values were $92.0 \pm 19.8 \mathrm{~g}$ prior to the intervention, and $95.2 \pm 17.5 \mathrm{~g}$ after the intervention. The comparison of the groups revealed that the stretching group had a significantly higher mean value than the control group prior to the intervention $(\mathrm{p}<0.01)$, and a significantly lower value than the control group after the intervention $(p<0.01)$. For the lateral hamstring, in the stretching group, the hardness values were $107.7 \pm 25.6 \mathrm{~g}$ prior to the intervention and 85.8 $\pm 14.9 \mathrm{~g}$ after the intervention, a significantly lower value after the intervention $(p<0.01)$. In the control group, the values were $97.3 \pm 24.1 \mathrm{~g}$ prior to the intervention and $96.6 \pm$ $19.7 \mathrm{~g}$ after the intervention. The comparison of the groups revealed the stretching group had a significantly higher mean value than the control group prior to the intervention $(\mathrm{p}<0.05)$, and a significantly lower value than the control group after the intervention $(\mathrm{p}<0.01)$.

Table 2 shows the results for leg rotation angle. Mean values and standard deviation of leg rotation angle are shown. In the stretching group prior to the intervention, the values were $0.97 \pm 1.82^{\circ}$ in the first phase, $0.21 \pm 4.94^{\circ}$ in the middle phase, and $1.99 \pm 6.74^{\circ}$ in the final phase. After intervention, the values were $1.30 \pm 3.31^{\circ}$ in the first phase, $1.01 \pm 6.34^{\circ}$ in the middle phase, and $3.58 \pm 7.24^{\circ}$ in the final phase. There was a significant increase in external rotation in the final phase following the intervention $(p<0.05)$. In the control group prior to intervention, the values were 1.53 $\pm 2.36^{\circ}$ in the first phase, $2.12 \pm 5.57^{\circ}$ in the middle phase, and $4.33 \pm 7.12^{\circ}$ in the final phase. After the intervention, the values were $1.29 \pm 2.16^{\circ}$ in the first phase, $1.23 \pm 4.83^{\circ}$ in the middle phase and $3.93 \pm 6.00^{\circ}$ in the final phase. No significant differences were found.

Table 3 shows the results for variation in leg rotation angle. Mean values and standard deviation of variation in leg 
Table 2. Changes in the leg rotation angle

\begin{tabular}{lllllll}
\hline & \multicolumn{3}{c}{ Stretching group } & \multicolumn{3}{c}{ Control group } \\
\cline { 2 - 7 } & First phase & Middle phase & Final phase & First phase & Middle phase & Final phase \\
\hline Pre-intervention (degree) & $0.97 \pm 1.82$ & $0.21 \pm 4.94$ & $1.99 \pm 6.74$ & $1.53 \pm 2.36$ & $2.12 \pm 5.57$ & $4.33 \pm 7.12$ \\
Post-intervention (degree) & $1.30 \pm 3.31$ & $1.01 \pm 6.34$ & $3.58 \pm 7.24^{*}$ & $1.29 \pm 2.16$ & $1.23 \pm 4.83$ & $3.93 \pm 6.00$ \\
\hline
\end{tabular}

Mean $\pm \mathrm{SD},{ }^{*} \mathrm{p}<0.05$, significant difference between pre- and post-intervention

Table 3. Changes in the variation in leg rotation angle

\begin{tabular}{lcc}
\hline & Stretching group & Control group \\
\hline Pre-intervention (degree) & $9.38 \pm 4.19^{\dagger}$ & $10.85 \pm 4.13$ \\
Post-intervention (degree) & $10.72 \pm 4.57^{*}$ & $10.01 \pm 3.67$ \\
\hline
\end{tabular}

Mean $\pm \mathrm{SD}, * \mathrm{p}<0.05$, significant difference between pre- and post-intervention

$\dagger \mathrm{p}<0.05$, significant difference between groups

Table 5. Changes in the variation in leg internal rotation angle

\begin{tabular}{lcc}
\hline & Stretching group & Control group \\
\hline Pre-intervention (degree) & $3.79 \pm 4.16$ & $2.91 \pm 3.28$ \\
Post-intervention (degree) & $3.63 \pm 4.15$ & $2.99 \pm 3.59$ \\
\hline
\end{tabular}

Mean \pm SD

rotation angle are shown. In the stretching group, the values were $9.38 \pm 4.19^{\circ}$ prior to the intervention and $10.72 \pm 4.57^{\circ}$ after the intervention, a significantly higher value after the intervention $(p<0.05)$. In the control group, the values were $10.85 \pm 4.13^{\circ}$ prior to the intervention and $10.01 \pm 3.67^{\circ}$ after the intervention. The comparison of the groups revealed the stretching group had a significantly lower value than the control group prior to the intervention $(\mathrm{p}<0.05)$, but no significant difference was found after the intervention.

Table 4 shows the results for variation in leg external rotation angle. Mean values and standard deviation of variation in leg external rotation angle are shown. In the stretching group, the values were $5.59 \pm 4.57^{\circ}$ prior to the intervention and $7.08 \pm 5.25^{\circ}$ after the intervention, a significantly value for the direction of external rotation after the intervention $(p<0.05)$. In the control group, the values were $7.94 \pm 5.73^{\circ}$ prior to the intervention and $7.02 \pm 4.66^{\circ}$ after the intervention. The comparison of the groups revealed the stretching group had a significantly lower value than the control group prior to the intervention $(\mathrm{p}<0.05)$, no significant difference was found after the intervention.

Table 5 shows the results for variation in leg internal rotation angle. Mean values and standard deviation of variation in leg internal rotation angle are shown. In the stretching group, the values were $3.79 \pm 4.16^{\circ}$ prior to the intervention and $3.63 \pm 4.15^{\circ}$ after the intervention. In the control group, the values were $2.91 \pm 3.28^{\circ}$ prior to the intervention and $2.99 \pm 3.59^{\circ}$ after the intervention. No significant difference was found between the groups, before or after the intervention.

Table 6 shows results for the muscle activities of the knee extensors. Mean values and standard deviation of $\% \mathrm{MVC}$
Table 4. Changes in the variation in leg external rotation angle

\begin{tabular}{lcc}
\hline & Stretching group & Control group \\
\hline Pre-intervention (degree) & $5.59 \pm 4.57^{\dagger}$ & $7.94 \pm 5.73$ \\
Post-intervention (degree) & $7.08 \pm 5.25^{*}$ & $7.02 \pm 4.66$ \\
\hline
\end{tabular}

Mean $\pm \mathrm{SD}, * \mathrm{p}<0.05$, significant difference between pre- and post-intervention

$\dagger \mathrm{p}<0.05$, significant difference between groups

Table 6. Changes in muscle activities of the knee extensors

\begin{tabular}{lllc}
\hline & & $\begin{array}{c}\text { Stretching } \\
\text { group }\end{array}$ & $\begin{array}{c}\text { Control } \\
\text { group }\end{array}$ \\
\hline Rectus & pre-intervention (\%) & $20.6 \pm 10.6$ & $24.0 \pm 12.4$ \\
femoris & post-intervention (\%) & $25.0 \pm 9.5^{*}$ & $24.7 \pm 10.5$ \\
\hline Vastus & pre-intervention (\%) & $24.8 \pm 12.7$ & $25.4 \pm 12.8$ \\
lateralis & post-intervention (\%) & $30.6 \pm 17.7^{*}$ & $28.1 \pm 12.6$ \\
\hline Vastus & pre-intervention (\%) & $24.0 \pm 12.4$ & $25.8 \pm 12.8$ \\
medialis & post-intervention (\%) & $25.9 \pm 13.2$ & $25.7 \pm 12.8$ \\
\hline
\end{tabular}

Mean \pm SD, ${ }^{*} \mathrm{p}<0.01$, significant difference between pre- and post-intervention

are shown. Muscle activities of the rectus femoris in the stretching group were $20.6 \pm 10.6 \%$ prior to the intervention and $25.0 \pm 9.5 \%$ after the intervention, a significantly higher value after the intervention compared to prior to the intervention $(p<0.01)$. In the control group, the values were 24.0 $\pm 12.4 \%$ prior to the intervention and $24.7 \pm 10.5 \%$ after the intervention. Muscle activities of the vastus lateralis in the stretching group were $24.8 \pm 12.7 \%$ prior to the intervention and $30.6 \pm 17.7 \%$ after the intervention, a significantly higher value after the intervention compared to prior to the intervention $(\mathrm{p}<0.01)$. In the control group, the values were $25.4 \pm 12.8 \%$ prior to the intervention and $28.1 \pm 12.6 \%$ after the intervention. Muscle activities of the vastus medialis in the stretching group were $24.0 \pm 12.4 \%$ prior to the intervention and $25.9 \pm 13.2 \%$ after the intervention. In the control group, the values were $25.8 \pm 12.8 \%$ prior to the intervention and $25.7 \pm 12.8 \%$ after the intervention.

Correlations between variation in muscle hardness and variation in leg rotation angle, variation in leg external rotation angle and variation in leg internal rotation angle are shown in Table 7. A moderate positive correlation was found between variation in leg rotation angle and muscle hardness of the lateral hamstring. A moderate positive correlation was found between the variations in leg external rotation angle and muscle hardness of the medial hamstring.

Correlations between variation in muscle hardness and 
Table 7. Correlations between variation in leg rotation angle and muscle hardness of the medial and lateral hamstrings

\begin{tabular}{lcc}
\hline & $\begin{array}{c}\text { Medial } \\
\text { hamstring }\end{array}$ & $\begin{array}{c}\text { Lateral } \\
\text { hamstring }\end{array}$ \\
\hline $\begin{array}{l}\text { Variation in leg rotation angle } \\
\text { Variation in leg external rotation }\end{array}$ & 0.21 & $0.36^{*}$ \\
$\begin{array}{l}\text { angle } \\
\text { Variation in leg internal rotation } \\
\text { angle }\end{array}$ & $-0.38^{*}$ & 0.28 \\
$* \mathrm{p}<0.01$ & & -0.11 \\
\hline
\end{tabular}

variations in muscle activities of the knee extensors are shown in Table 8. No significant correlations were found between variations in muscle hardness of the medial and lateral hamstrings, and variations in the muscle activities of the rectus femoris, vastus lateralis and vastus medialis.

\section{DISCUSSION}

Knee extension is often conducted as a therapeutic exercise for knee joint dysfunction, and depending on the remaining muscular strength of the quadriceps femoris muscles active resistive exercise, active exercise, active assistive exercise, or passive exercise is selected ${ }^{1}$. Since each type of motion involves mechanical load being applied to the knee joint, which serves as a fulcrum, it is important that the joint motion of the knee be kinematically proper when knee extension is conducted. In knee joint motion, leg rotation accompanies flexion and extension ${ }^{2-4)}$. Thus, in knee extension as a therapeutic exercise for knee joint dysfunction, it is necessary that knee joint motion should be kinematically proper during exercise. However, many studies have analyzed leg rotation ${ }^{7-13)}$, there have been few studies of the causes of the failure of leg rotation. Focusing on the hamstrings, the antagonists of knee extension, whether the extensibility of the hamstring was a limiting factor of leg rotation was investigated by examining the effect of change in the extensibility of the hamstring on leg rotation during knee extension. To do this, leg rotation was measured during knee extension before and after stretching of the hamstring and factors correlating with this, including muscle activities of the knee extensors, were investigated.

One method for checking extensibility of the hamstring is the straight leg raise angle. However, as this is a measurement that involves joint motion, it includes changes in the flexibility of the joint in addition to changes in the extensibility of the muscles. Since the subject of evaluation in this study was the joint itself, we used a muscle durometer which examines just the hardness of the muscles. The results of the muscle hardness measurements show that the muscle hardness of the stretching group was significantly higher than that of the control group before the intervention, and this is thought to because the selected subjects had higher muscular hardness of the medial hamstring in the initial measurement. Muscle hardness of the lateral hamstring on the same side was also significantly higher than that of the control group. Muscle hardness can be observed in the body motion
Table 8. Correlations between variation in muscle hardness of the medial and lateral hamstrings and variations in the muscle activities of the rectus femoris, vastus lateralis and vastus medialis

\begin{tabular}{ccc}
\hline & $\begin{array}{c}\text { Medial } \\
\text { hamstring }\end{array}$ & $\begin{array}{c}\text { Lateral } \\
\text { hamstring }\end{array}$ \\
\hline Rectus femoris & 0.004 & 0.080 \\
Vastus lateralis & 0.189 & 0.005 \\
Vastus medialis & 0.115 & -0.024 \\
\hline
\end{tabular}

in daily activities and in a range of sports. Laterality of center of gravity during motion arises since there is laterality and non-laterality in bodily movements, such as bilaterally simultaneous actions like standing-up and squatting, or alternate movement as in walking. Thus, laterality arises in muscular activity during motion, and laterality in muscle hardness may also arise without the subject's awareness. From this we can infer that muscle hardness may also have been high in the lateral hamstring of the stretching group which exhibited significantly high muscle hardness of the medial hamstring.

Straight leg raises are normally used as a method for stretching the hamstring, but the stretching force applied to the hamstring passes through the hip and knee joints in this case and this stretching force may change the flexibility of these joints. In this study we conducted direct stretching, the effects of which have been reported to be measurable by the finger-floor distance ${ }^{16)}$, since it was important that the hamstrings were stretched without affectiong the joints, which were the target of evaluation. As a result of this intervention, muscle hardness was significantly reduced in the medial and lateral hamstrings of the stretching group in which pre-intervention muscle hardness was significantly high. This indicates that the extensibility of the hamstring had increased, with post-intervention active knee extension probably conducted in a state of high extensibility of the hamstring.

The causes of leg rotation in the direction of external rotation during knee extension exercises are divided into passive and active factors. In passive exercise, differences in the size of the medial and lateral condyles of the femur and strain in the anterior cruciate ligament act as passive factors. When there is extension from a state of knee flexion, the articular surface of the tibia shifts to the anterior, following the medial and lateral condyle of the femur. However, since the articular facet of the medial condyle of the femur is larger than the articular facet of the lateral condyle of the femur, the anterior migration length of the contact point with the articular surface of the tibia becomes longer in the medial condyle of the femur than in the lateral condyle of the femur. At 90 degrees of flexion, the contact point with the medial condyle of the femur is situated in a more posterior position. Subsequently, while these anterior shifts of contact points occur in parallel, leg rotation occurs in the direction of external rotation since the contact point with the medial condyle of the femur shifts more to the anterior than the lateral condyle of the femur from 30 degrees of 
flexion to full extension. Also strain in the anterior cruciate ligament increases with extension, inducing anterior shift of the tibia. This strain reaches its maximum point at full extension, causing traction in the direction of external rotation. In active assistive exercise and active exercise, in addition to the passive factors, external traction against the tibia induced by the muscular strength of the quadriceps femoris muscle is added as an active factor. When quadriceps femoris knee extension exercises are conducted, the vastus muscle group provides $80 \%$ of the work, and the other $20 \%$ is provided by the rectus femoris muscle ${ }^{2)}$. The vastus lateralis muscle has the widest cross-sectional area in the vastus muscle group. Thus, the traction direction in relation to the patella of the quadriceps femoris muscle generally tends to be in the external direction, causing traction in the direction of external rotation in leg rotation. Limiting factors in leg external rotation may include deformation of the medial and lateral condyles of the tibia, dysfunction and damage of the anterior cruciate ligament, and decreased strength of the quadriceps femoris muscle. However, all of the subjects in this study were young, healthy individuals without a history of articular disease. Therefore, factors such as bone deformation, dysfunction and damage of the anterior cruciate ligament, and decreased strength of the quadriceps femoris muscle should have been absent in this group. Ishii et al. ${ }^{17)}$ reported multiple patterns of leg rotation in healthy individuals, which they explained were due to "general joint laxity." It is possible that decreased extensibility of the hamstrings might be one factor of general joint laxity. When extensibility of the hamstring decreases, active knee extension is conducted with the force from the head of the fibula being pulled in the posterior direction. This is due to the head of the fibula being in close contact with the tibia and proximal tibia which is the insertion point for the semitendinous, semimembraneous and biceps femoris muscles, which form the hamstring. Thus, the anterior shifts of the medial and lateral condyles of the tibia are limited, the proximal tibia in the region of full knee extension falls in the posterior direction, and the proximal end of the tibia cannot reach a sufficiently anterior position. As the articular surface of the tibia shifting anteriorly to follow the medial and lateral condyle of the femur becomes insufficient, strain in the anterior cruciate ligament does not increase in the region of full knee extension. This limits leg rotation in the direction of external rotation. Hence, prior to the intervention when the extensibility of the hamstring was comparatively low, the angle of leg rotation, variation in leg external rotation angle, and variation in leg internal rotation angle exhibited low values. However the restriction of the anterior shift of the contact point of the medial condyle of the tibia with the medial condyle of the femur in the region of full knee extension disappeared as extensibility of the hamstring increased following the intervention. Because natural leg rotation in the direction of external rotation becomes possible at this point, the angle of leg rotation, variation in leg external rotation angle, and variation in leg internal rotation in the final phase of extension significantly increased. The increase in the angle of leg rotation as extensibility of the hamstring increased is supported by the moderately positive correla- tion that was found between the variation in muscle hardness of the lateral hamstring and the variation in leg rotation angle, and between the variation in muscle hardness of the medial hamstring and the variation in leg external rotation angle. These findings indicate that reduction in extensibility of the hamstring may be a limiting factor for leg rotation.

In terms of activity of the quadriceps femoris muscle during active knee extension, no correlation was found between change in muscle hardness of the hamstring due to the intervention and change in the activity of the quadriceps femoris muscle. However, the activity of the quadriceps femoris muscle increased despite the subject being in the supine position, in which active knee extension is not as easily affected by the hamstring. Nakajima et al. ${ }^{18)}$ reported that stretching of the hamstring increased knee extension torque during uniform motion at an angular velocity of $180 \%$ sec. Their research did not use electromyography, however their results mirror ours in that the increased extension torque resulting from increased muscle activity suggests the activity of the quadriceps femoris muscle increased due to hamstring stretching. A possible explanation for this is that, generally when a muscle elongates, a response to the tensile stimulation occurs in the intramuscular muscle spindle. When this surpasses optimal strength, a stretch reflex is produced in the same muscle, and the antagonist is suppressed. When there is gamma motor neuron activity, the receptor site in the intrafusal muscle fiber expands due to this activity ${ }^{19}$. Thus, stretch reflex increases on account of the increased frequency of responses generated from the muscle spindle. Therefore, when the hamstring elongates from resting length due to knee extension, prior to intervention when extensibility of the hamstring wsa reduced, the muscle elongates in a way that triggers the gamma motor neuron activity which regulates the length of the intrafusal muscle fiber in response to muscle extensibility. Thus, the response frequency of the muscle spindle increases, increasing the stretch reflex, triggering a suppressive effect on the quadriceps femoris muscle which has an antagonistic relationship, thus reducing that activity. After the intervention, however, the extensibility of the hamstring had increased. This in turn reduced gamma motor neuron activity, which regulates the length of the intrafusal muscle fiber, reducing the response frequency of the muscle spindle, and decreasing the stretch reflex. Accordingly, there was a decrease in the suppressive effect acting on the quadriceps femoris muscle, which increased the activity of that muscle. As an active factor causing leg rotation during knee extension, the quadriceps femoris muscle exerts traction against the tibia in the external direction ${ }^{2}$. Thus, the angle of leg rotation, variation in leg external rotation angle, and variation in leg internal rotation increased in the final phase of the extension due to this increased activity.

The present results indicate that reduced extensibility due to stretching of the hamstring affects the anterior shift of the tibia along with the femoral shape, which is a passive factor in leg rotation. In addition, it affects the activity of the quadriceps femoris muscle, which is an active factor. The present findings indicate that when conducting knee extension as a therapeutic exercise for dysfunction of the 
knee, extensibility of the hamstring and leg rotation should be evaluated. When proper leg rotation is not found, extensibility should be increased by hamstring stretching, to restore the proper leg rotation associated with active knee extension. This would not affect patients with abnormal structural factors, including athletes who have suffered anterior cruciate ligament injury or knee osteoarthritis patients with clear bone deformity. Increasing extensibility of the hamstring by stretching would also be effective as a pretreatment for restoring proper leg rotation when knee extension is conducted as a therapeutic exercise for athletes who have not suffered anterior cruciate ligament injury and knee osteoarthritis patients initially lacking clear bone deformity.

\section{ACKNOWLEDGEMENTS}

I am deeply grateful to Professor Yasusuke Hirasawa and Associate Professor Kazuhisa Matsumoto, Department of Rehabilitation, Meiji University of Integrative Medicine, who provided carefully considered feedback and valuable comments. I also thank Masanori Kakimura, Yoshiaki Kanda, Aiko Hayashi, Rintaro Konishi, Tomotaka Nagayama and Kenichi Tomita, Rehabilitation Center, Meiji University of Integrative Medicine Hospital, for their technical assistance with this study. I also thank many students who belong to Meiji University of Integrative Medicine for their cooperation.

\section{REFERENCES}

1) Rusk HA: Principles of Physical Therapy. In: Rehabilitation Medicine Saint Louis: The C.V. Mosby Company, 1977, pp 93-122.

2) Donald AN: Knee. In: Kinesiology of the Musculoskeletal System; Foundation for Rehabilitaion. United States: Mosby Elsevier, 2010, pp 520-572.
3) Hollister AM, Jatana $S$, Singh AK, et al : The axes of rotation of the knee Clin Orthop Relat Res, 1993, 290: 259-268. [Medline]

4) Straiton JA, Todd B, Venner RM: Radiographic assessment of knee joint rotation. J Anat, 1987, 155: 189-193. [Medline]

5) Hallén LG, Lindahl O: The "screw-home" movement in the knee-joint. Acta Orthop Scand, 1966, 37: 97-106. [Medline] [CrossRef]

6) Sugawa S, Kito N, Shimazawa S, et al.: Analysis of femoral leg rotation movement during walking among patients with medial knee osteoarthritis. J Jpn Phys Ther Assoc, 2004, 31: 412-419.

7) Nagao N, Tachibana T, Mizuno K: The rotational angle in oeteoarthritic knees. Int Orthop, 1998, 22: 282-287. [Medline] [CrossRef]

8) Hamai $\mathrm{S}$, Morooka $\mathrm{T}$, Miura $\mathrm{H}$, et al.: Knee kinematics in medial osteoarthritis during in vivo weight-bearing activities. J Orthop Res, 2009, $1555-1560$.

9) Koga T, Ohmori T, Suzuki T, et al.: Motion analysis on the pathogenesis of knee osteoarthritis. Jpn J Surg, 1997, 16: 327-333.

10) Ishii $T$ : The screw home movement become unclear with progression of stage of OA knee. Niigata Igakkai Zasshi, 1999, 113: 304-314.

11) Kuriwaka Y: A biomechanical study in the osteoarthritis of the knee - particularly, rotatory movement of knee joint - . J Jpn Orthop Assoc, 1982, 56 : 713-726.

12) Nagao N, Tachibana T, Mizuno K: Rotatory movement of osteoarthritic knee by ultrasonography. Cent Jpn J Orthop Surg Traumatol, 1995, 38: 1641-1642.

13) Nagao N, Tachibana T, Yokoi A, et al.: Rotation of the osteoarthritic knee by ultrasonography-The second report. Cent Jpn J Orthop Surg Traumatol, 1996, 39: 1493-1494.

14) Edward FD, Aldo P: Thigh. In: Anatomic Guide for the Electromyographer; The Limbs. Niigata: Nishimura Co Ltd, 1985, pp 164-195.

15) Matsumoto K, Kimura A, Tomita K, et al.: Effect of meridian and muscle region stretching on alleviation of delayed onset muscle soreness. 14th International Congress of the World Confederation for Physical Therapy-CD of Abstracts-, 2003: RR-PO-0672.

16) Ohtsuki K, Suzuki T: A comparison of the immediate changes in subjects with chronic lower back pain effected by lower back pain exercises and direct stretching of the tensor fasciae latae, the hamstring and the adductor magnus. J Phys Ther Sci, 2012, 24: 97-100. [CrossRef]

17) Ishii $S$, Yamamoto $S$ : Kinematic analysis of screw home movement with active knee extension in the non-weight bearing position. Rigakuryoho Kagaku, 2008, 23: 11-16. [CrossRef]

18) Nakajima R, Hirano $D$, Akazawa $K$, et al.: Does improvement of hamstring tightness infuluence knee extensor strength? Rigakuryoho Kagaku, 2012, 27: 51-54. [CrossRef]

19) Itoh F: Kinbousui (Ia, IIgun) Kankakushingou no Naiyou. In: Kinkankakukenkyu no Tenkai. Tokyo: Kyodo Isho Shuppan, 2005, pp 253-281. 